\title{
A Modelica Contact Library for Idealized Simulation of Independently Defined Contact Surfaces
}

\author{
Felix Oestersötebier \\ Peng Wang \\ Ansgar Trächtler \\ Control Engineering and Mechatronics, Heinz Nixdorf Institute, University of Paderborn \\ Fürstenallee 11, 33102 Paderborn, Germany \\ \{felix.oestersoetebier, peng.wang, ansgar.traechtler\}@hni.uni-paderborn.de
}

\begin{abstract}
Modeling of multibody mechanics plays a central role in the design of mechatronic systems. In technical use-cases, they often contain loose couplings, where contact is possible. We present a ready-to-use contact library in Modelica. It comprises surface definitions for simple contact surfaces, which can be connected with multibodies of the Modelica Standard Library. It implements a force-based approach between single contact points. The contact forces are calculated in configurable non-central contact blocks. Exemplarily, the results of three experiments are shown and compared to benchmark simulations.
\end{abstract}

Keywords: contact library; simple contact surfaces; non-central contact block; contact forces

\section{Introduction and Motivation}

In the design process of mechatronic systems, the designers are facing the challenge of developing and controlling the more and more complex dynamics of the system. Therefore, multi-domain simulation models come to use from the outset of the conceptual design stage. As this also denotes a significant modeling effort, object-oriented modeling languages like Modelica offer the possibility to utilize and/or build up model libraries. The concept of ports makes it possible to combine and simulate components of different domains and origins in one model of the system. We intend to extend the available libraries by providing an idealized contact library that makes it possible to model contact phenomena. In particular, it should be possible to define each component separately in order to be able to reuse and combine approved patterns in new applications (c.f. [1]).

Considering technical use-cases one often finds multibody mechanics that typically comprise mainly fixed (e.g. kinematic chains) and few loose couplings, where dynamic contact phenomena take place. Nevertheless, modeling contacts is "a key factor and a challenging problem in simulation of multibody systems (MBS), where a balance between performance and accuracy has to be found" [2]. However, to the best of our knowledge, there is currently no ready-to-use Modelica library available to handle contact problems in any level of detail. Otter et al. [3] suggest a force-based extension to the Modelica MultiBody Library to enable central collision handling. Herein, three variants to define contact surfaces are described (parametric surfaces, algebraic constraint surfaces, surfaces of polytopes). In [4] the surfaces of arbitrary bodies in the MBS are discretized by means of polygons. The approach presented in this paper differs from that, as it implements contact modeling by means of noncentral contact blocks. It provides combinable, simple contact surfaces, which are described by single contact points.

We observed that in many industrial applications, even if the complete bodies are of complex shape, only a certain part of them contacts with others. The actual contact surfaces are often designed to be simple. Thus, the aim is to enable the designer to perform simulations of such systems including idealized representation of the contacts. Thereby, the analysis of the principle functional capability of the system in the course of the conceptual design is focused. This entails specialized modeling principles concerning the usability and the interpretation of the simulation results, which both should be relatively easy.

\section{Concept of the Contact Library}

Multibody mechanics is usually modeled using rigid bodies, which are described by their mass, located in the center of mass $(\mathrm{CM})$, and their moment of inertia. In order to be able to model elastic, non-stiff collisions one has to weaken the rigid body assumption a little. Furthermore, one has to consider 
the shape of the bodies. We address these two issues in the following subsections.

\subsection{Idealization of the Elastic Foundation Model}

References in literature introduce a so called "foundation model" to approximate the complex contact theory of Hertz and others in the context of multibody simulation $[4,5,6,7,8]$. It is assumed that the contact area is small compared to the dimensions of the contacting bodies (nonconforming contacts). The foundation model comprises a thin elastic or viscoelastic layer between the rigid bodies and neglects shear stress. With these assumptions, which are valid for isotropic and homogenous materials, one is able to describe dynamic as well as static contact incidents. The force-based approach contrasts with idealized impulse-based calculations, which are only applicable for stiff contacts [3].

We use a further idealization (c.f. Figure 1) that is based on single force elements. Assuming that the contact area is not only relatively small but also of an idealized shape, we describe it by means of single contact points. A nonlinear spring-damper element is inserted to calculate the normal force $F_{n}$ between these points of the colliding surfaces. This requires the previous identification of possible contact points on the rigid body surfaces and the continuous determination of the normal direction. For these purposes, we provide analytic solutions for simple geometries in our library. The normal force is then used to determine the friction force $F_{t}$ between the two bodies in the tangential direction.
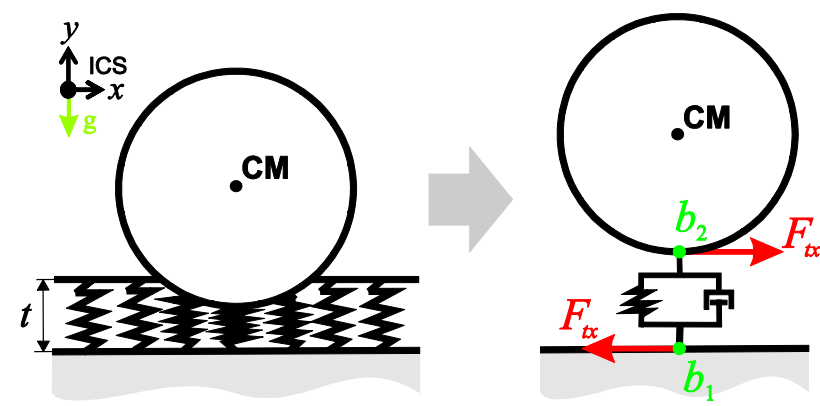

Figure 1: Idealization of the elastic layer

\subsection{Classification of Contacts}

As a starting point, we focused on spherical, cylindrical and plane surfaces, either in rectangular or circular shape. Depending on the shape of the contact area, we use 1 (punctiform), 2 (linear) or 4-5 (planar) points to describe it. The position of the respective number of potential contact points is calculated on both of the contact bodies and the collision detection is performed for each pair of potential contact points. Figure 2 shows the shape of the contact area for all possible combinations, as well as the number of contact points. As the contact region may alter with the moving bodies, the contact points will also move on the defined surface.

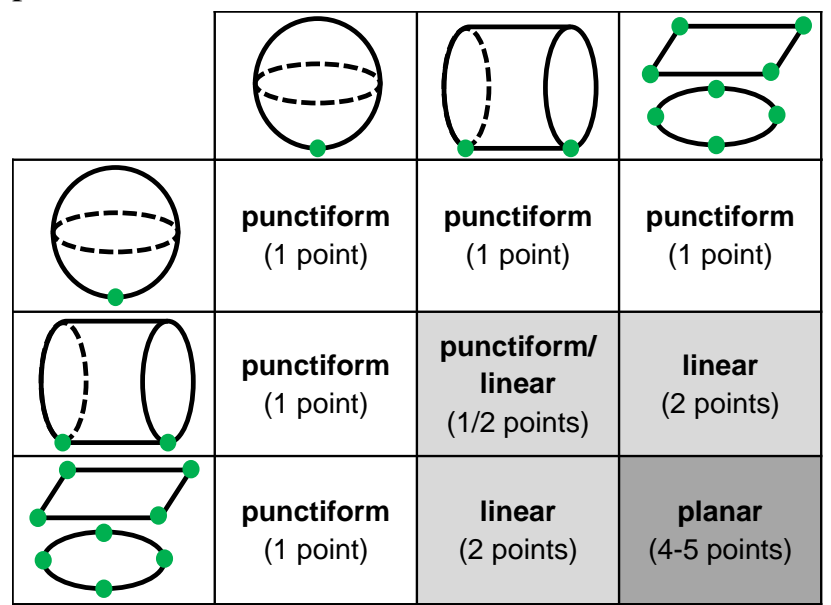

Figure 2: Shapes of the contact area and number of contact points for the different contact pairs ${ }^{1}$.

\subsection{Definition of Contact Surfaces}

To describe the aforementioned, elementary contact surfaces (Figure 2), we provide ready-to-use blocks. The surface blocks represent a thin and massless layer, which can be connected to any kind of rigid body by a frame connector (c.f. Figure 3). The dimensions of the surface can be parameterized. In order to be able to use the dimensions in the contact block we introduce a new interface to connect the surface definition with a contact block. This adds a vector to the MultiBody frame of the Modelica Standard Library that comprises maximum three terms to describe the surface geometry (e.g. width, length) and the respective direction vectors to get the orientation in the connected frame. The latter constitutes the body coordinate system (BCS) of the contact surface.

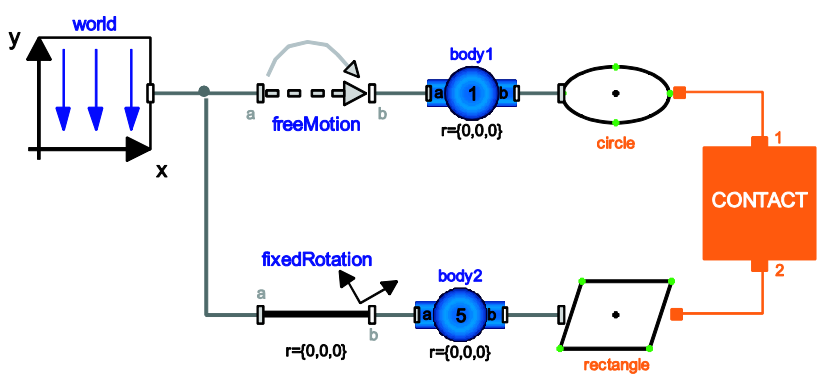

Figure 3: Example of a multibody model including contact

\footnotetext{
${ }^{1}$ Spherical, cylindrical and plane surfaces are displayed by the respective icon, which also indicates the maximum number of contact points.
} 
Figure 3 displays an example of the intended use of the contact library. While the shape of the rigid bodies may be arbitrary, the shapes of the surfaces that possibly can collide are defined to be a circular and a rectangular plane. For example, body 1 may represent a bottle with a round and plane base. As this stands on a rectangular desk only these two simple contact surfaces have to be defined. More complex contact surfaces may be assembled from elementary ones, where every pair of surfaces is connected via a contact block. The contact block is adjusted to the respective surface combination by using the replaceable method for class parameterization in Modelica.

\section{Modeling of the Contact Block}

The calculation of the appropriate force in the contact block clearly depends on the combination of surfaces. Nevertheless, a comprehensive sequence (c.f. Figure 4) can be established that is implemented in the contact block and performed in each time step. As mentioned before, the sphere-to-sphere, sphereto-cylinder and sphere-to-plane contact areas are described via a punctiform contact. In these cases, three steps have to be passed to determine the contact force. First, a potential contact point is determined on each of the two contact surfaces. The contact condition for these two points is checked in the next step. If the condition is fulfilled, the two bodies collide and the contact force between the two contact points is calculated. Otherwise, no contact force is applied.

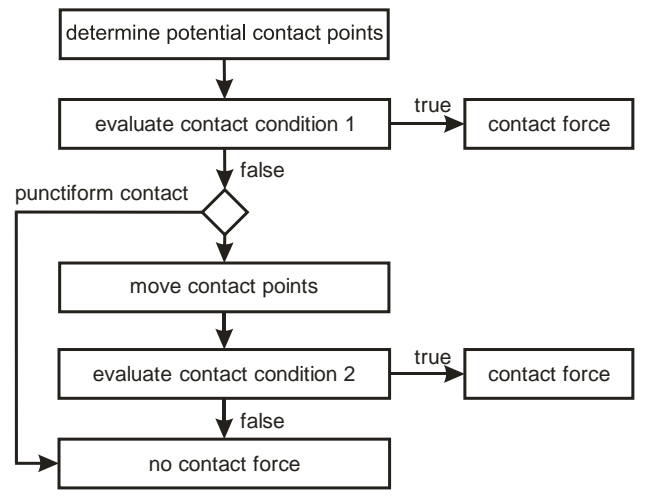

Figure 4: Calculation of the contact force

In the case of linear or plane contact, the contact area may become smaller if only parts of the surfaces collide, whereas the shape stays the same. Thus, additional contact point movement may be necessary. Here, the detection of contact points and the contact condition checking are performed analogously in the first two steps. If the contact condition 1 is not fulfilled, the contact points are displaced to a new position on the contact surfaces in a way that contact between the new potential contact points might be possible. The latter is checked by evaluating the second contact condition. If a collision occurs, the respective contact force is calculated and applied.

\subsection{Contact Detection}

The main principle of the implemented contact detection is shown with the help of two examples. The first example comprises a contact between a sphere and a cylinder (c.f. Figure 5). Initially, the bodies are represented by their centroids $a_{1}$ and $a_{2}$ in the MBS model. Two body-fixed frames BCS1 and BCS2 describe these two points. Furthermore, we assume that the lateral surface of the cylinder and the spherical surface of the ball are defined according to Section 2.3. Consequently, the two frames, the radius $r$ of the ball and the length $L$ and the diameter $D$ of the cylinder, as well as the direction vectors of $L$ and $D$ resolved in BCS1, are available in the contact block.

In order to determine the potential contact points, the sphere-centroid $a_{2}$ is projected on the longitudinal direction of the cylinder. The latter is represented by the $x$-direction of BCS1 by default. The result of the projection is the point $a_{2}^{\prime}$ given in BCS1. However, the absolute position of the point $a_{2}^{\prime}$ is limited to $\pm L / 2$. This ensures that the point is always located between the two end faces of the cylinder. The local coordinate system LCS1 is defined in $a_{2}^{\prime}$ and has the same orientation as BCS1.

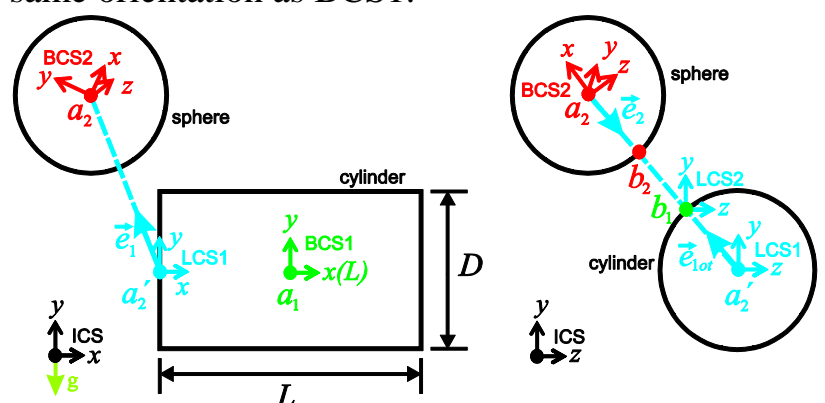

Figure 5: Contact point detection of the sphere-tocylinder contact model

Based on vector $\overrightarrow{a_{2}^{\prime} a_{2}}$ the direction vector $\vec{e}_{1}$ is computed. Then, the orthogonal projection $\vec{e}_{1 o t}$ of the vector $\vec{e}_{1}$ is determined in the $y$-z-plane of LCS1. We obtain the first potential contact point $b_{1}$ by displacing $a_{2}^{\prime}$ along the vector $\vec{e}_{10 t}$. As $b_{1}$ lies on the cylinder surface, the distance is given by $D / 2$. In the potential contact point $b_{1}$, the local coordinate system LCS2 is defined. Again, the orientation of it is equivalent to BCS1. With the help of LCS2 the position of the potential contact point $b_{2}$ on the ball- 
surface can be calculated. Therefore, the vector $\overrightarrow{a_{2} b_{1}}$ and the direction vector $\vec{e}_{2}$ are determined. By moving $a_{2}$ along $r \vec{e}_{2}$ we calculate the position of the second potential contact point $b_{2}$ on the spherical surface. The vector $\vec{e}_{2}$ also constitutes the normal direction for the force calculation (c.f. Section 3.3) in this case.

After the determination of the potential contact points $b_{1}$ and $b_{2}$, the contact condition is evaluated (c.f. Equation (1)). It consists of two terms. On the one hand, it is verified that the distance between the points $b_{2}$ and $a_{2}^{\prime}$ is less than radius of the cylinder end face $(D / 2)$ and greater than the difference between the radius and the maximum penetration $t$. The latter results from the assumption of a thin contact layer. This evaluation is performed in LCS1. On the other hand, the interval $\left|x_{b_{2}}\right|$ between $b_{2}$ and $a_{1}$ in the longitudinal direction of the cylinder has to be less than or equal to $L / 2$. This is evaluated in BCS1. If both conditions are fulfilled at the same time, the two bodies intrude and the contact force is applied to the contact points (c.f. Section 3.3).

$$
\begin{aligned}
& \text { contact }=\left(\frac{D}{2}-t \leq\left|\overrightarrow{a_{2}^{\prime} b_{2}}\right| \leq \frac{D}{2}\right) \\
& \cap\left(\left|\vec{e}_{x B C S 1} \cdot \overrightarrow{a_{1} b_{2}}\right|=\left|x_{b_{2}}\right| \leq \frac{L}{2}\right)
\end{aligned}
$$

The second example is the collision between a cylinder and a circular plane (see Figure 6), which denotes a linear shape of the contact area. As regards the circular plane, the geometry is sufficiently described by the radius $R$. Again, body-fixed coordinate systems (BCS1 and BCS2) are defined in the centroids $a_{1}$ of the cylinder and $a_{2}$ of the plane. The $y$-direction of BCS2 represents the vertical direction of the plane and the normal direction for force calculation. According to Figure 2 the contact area has a linear shape. Thus, two potential contact points have to be determined on the surface of the cylinder. These do not necessarily touch the plane at the same time.

To detect the contact points on the surface of the cylinder, the centers $a_{1 l}$ and $a_{1 r}$ of the two end faces are observed. The local coordinate systems LCS1 and LCS2 in these points are oriented like BCS2. In preparation for the potential contact point $b_{11}$, the unit vector $\vec{e}_{L}$ of the $x$-direction in BCS1 and the orthogonal projection $\vec{e}_{L p}$ on the $x$ - $z$-plane of BCS2 are determined. Then, we implemented the following rotation sequence to get local coordinate systems that are orientated as needed. As regards $a_{1 l}$, the local coordinate system LCS1 is rotated about the $y$-axis (LCS'1) and the $x$-axis (LCS"1) thereafter. The rotation angle $\alpha$ is determined by the scalar product of $\vec{e}_{L p}$ and the unit vector of the $x$-direction of the
BCS2. Whereas $\beta$ is specified by the angle between the vectors $\vec{e}_{L}$ and $\vec{e}_{L p}$. The contact point $b_{11}$ is then given by $(0,-D / 2,0)$ in the obtained system LCS"1. With the same principle, one can also determine the point $b_{12}$. In addition, the contact points $b_{21}$ and $b_{22}$ on the surface of the circular plane can be located by projecting $b_{11}$ and $b_{12}$ onto the plate (BCS2).

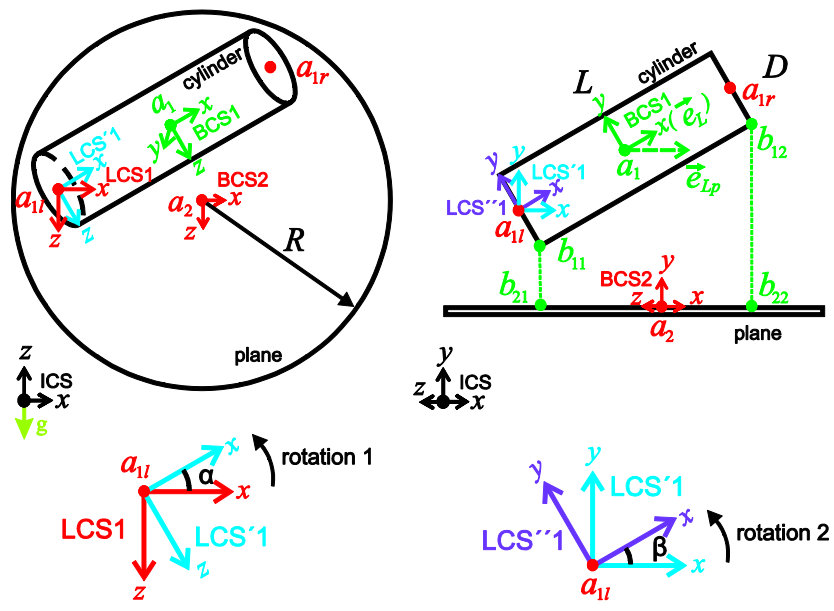

Figure 6: Contact point detection of the circle-tocylinder contact model

Based on these preliminary, potential contact points the contact condition 1 is evaluated. It consists of three parts. As an example, the condition related to $b_{11}$ is given in Equation (2). The variables $x_{b_{11}}, y_{b_{11}}$ and $z_{b_{11}}$ are the components of $b_{11}$ in the corresponding direction given in BCS2. To detect a collision, the absolute position in the $x$ - and $z$ direction must not be greater than the radius of the plane, and the penetration depth must not exceed the maximum value of $t$ in the negative $y$-direction.

$$
\begin{aligned}
\text { contact } 1= & \underbrace{\left(\left|x_{b_{11}}\right| \leq R\right)}_{i} \cap \underbrace{\left(-t \leq y_{b_{11}} \leq 0\right)}_{i i} \\
& \underbrace{\left(\left|z_{b_{11}}\right| \leq R\right)}_{\text {iii }}
\end{aligned}
$$

\subsection{Contact Point Movement}

However, contact between the cylinder and the circular plane might be possible, even if the contact condition 1 is not fulfilled. Figure 7 shows a configuration where the term $i$ in Equation (2) is false. In this case, the preliminary contact point $b_{11}$ should be displaced along the contact surface. We use the law of cosines to calculate the position of this new point $b^{\prime}{ }_{11}$. Considering the displayed triangle (Figure 7), we get the displacement $b$ by the following equations.

$$
\begin{gathered}
R^{2}=a^{2}+b^{2}-2 a b \cdot \cos \gamma \\
a=\left|\overrightarrow{b_{11} a_{2}}\right| \\
\gamma=\measuredangle\left(\overrightarrow{b_{11} a_{2}}, \overrightarrow{b_{11} b_{12}}\right)
\end{gathered}
$$




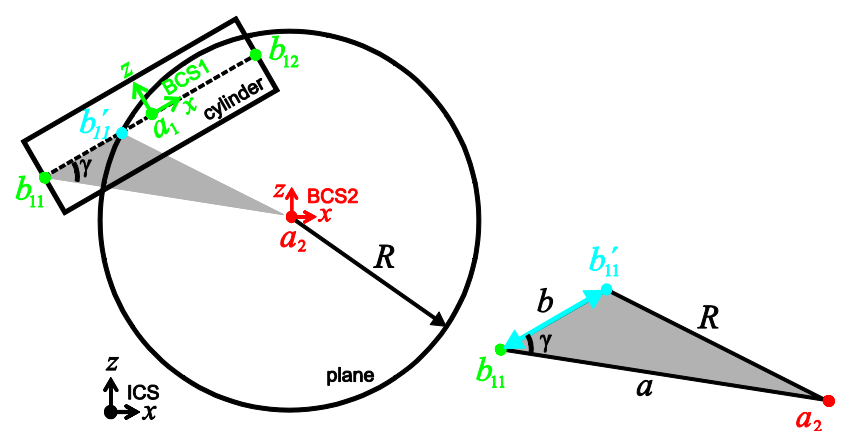

Figure 7: Contact point movement in the circle-tocylinder contact model

The vector $\overrightarrow{b_{11} b_{12}}$ can be replaced by the length direction of the cylinder, which is available from the contact interface (c.f. Section 2.3). Thus, the new contact point $b_{11}^{\prime}$ is determined and the analogous contact condition 2 is checked.

$$
\begin{aligned}
\text { contact } 2= & \left(\left|x_{b_{11}^{\prime}}\right| \leq R\right) \cap\left(-t \leq y_{b_{11}^{\prime}} \leq 0\right) \\
& \cap\left(\left|z_{b_{11}^{\prime}}\right| \leq R\right)
\end{aligned}
$$

Since every linear contact region denotes a possible one-directional displacement of preliminary contact points, the contact detection in these cases is very similar. In contrast, planar contact regions lead to two directions of motion. Exemplarily, the contact point movement in the case of two rectangular planes is described in the following.

The initial situation of the example is shown in Figure 8. Again, the body-fixed coordinate systems BCS1 and BCS2 are defined in the two centers $a_{1}$ and $a_{2}$. The geometry information of each plane contains the length $L$ and the width $W$. The potential contact points are placed in the four corners of the planes. The contact condition in Equation (5) is used for the potential contact point $b_{11}$.

$$
\begin{aligned}
\text { contact } 1= & \underbrace{\left(\left|x_{b_{11}}\right| \leq \frac{L_{2}}{2}\right)}_{i} \cap \underbrace{\left(-t \leq y_{b_{11}} \leq 0\right)}_{i i} \\
& \cap \underbrace{\left(\left|z_{b_{11}}\right| \leq \frac{W_{2}}{2}\right)}
\end{aligned}
$$

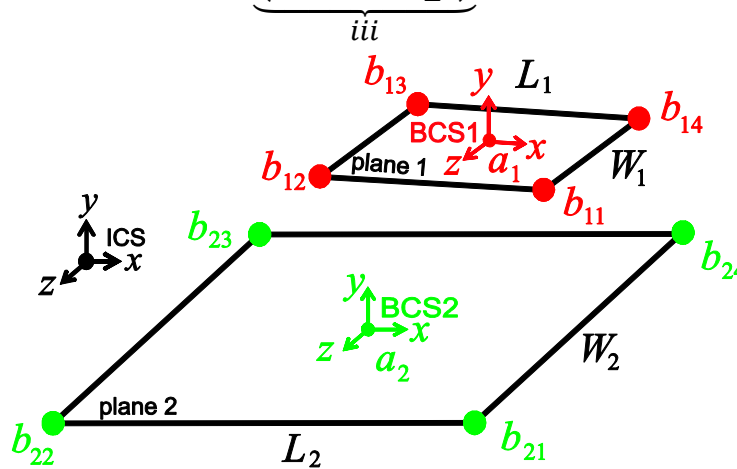

Figure 8: Initial contact points and coordinate systems of the rectangle-to-rectangle contact model

If the contact condition in Equation (5) cannot be fully met, the corresponding point is displaced along the two adjacent edges of the rectangular plane and two new points are obtained. However, it must be ensured that the displacement cannot exceed the length of the respective edge. Figure 9 exemplarily shows a configuration, where one of the initial contact points of plane 1 is outside plane 2. The condition $i$ in Equation (5) is violated, which means that the contact point exceeds plane 2 in $x$-direction of BCS2. The height $h_{o l}$ of the displayed triangle constitutes the distance between $b_{11}$ and the nearest edge on plane 2. It is calculated by the following equation.

$$
h_{o l}=\left|x_{b_{11}}\right|-\frac{L_{2}}{2}
$$

The relationship between the displacement of $b_{11}$ and the distance $h_{o l}$ can then be expressed with the help of the angle $\varphi$.

$$
\begin{aligned}
V_{l w} & =\min \left(\frac{h_{o l}}{|\sin \varphi|}, W_{1}\right) \\
V_{l l} & =\min \left(\frac{h_{o l}}{|\cos \varphi|}, L_{1}\right)
\end{aligned}
$$

From these equations the two new potential contact points $b_{11 l}$ and $b_{11 w}$ are obtained by circumventing singularity problems in case of $\varphi=0$ or $\varphi=\pi / 2$. Again, the second collision detection is evaluated to check whether contact forces have to be applied or not. As a result, it is possible that a maximum of five points can represent planar contact areas.

When implementing the contact point movement in Modelica, we tried to minimize the number of DAEs and events. Therefore, for example the prismatic block of the Modelica Standard Library has been modified in order to be adaptable to the respective direction. It is furthermore possible to disable the inserted filtering of the contact point displacement. Because it is usually continuously differentiable, this denotes an effective possibility to reduce the simulation time very often (see Section 4).

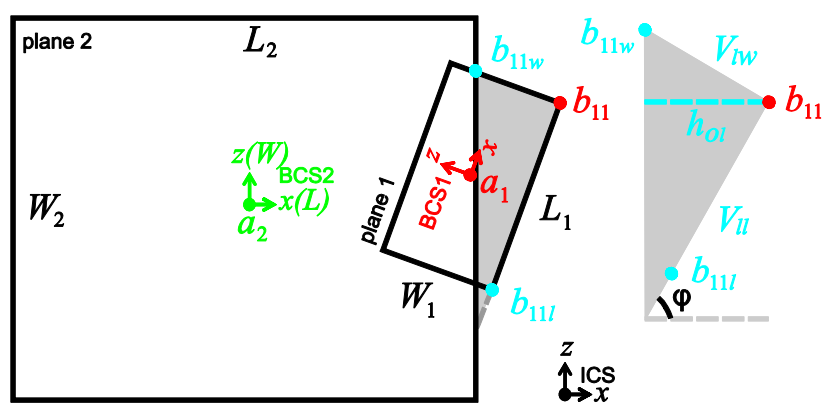

Figure 9: Contact point movement of the rectangleto-rectangle contact model

\subsection{Contact Forces}

The three-dimensional contact force is applied, if the contact condition holds for one contact point. It 
consists of both the normal force and the tangential friction force. The respective directions can be obtained by means of the local coordinate systems in the contact points. As aforementioned, the continuous surface layer is replaced by a nonlinear spring-damper element. Consequently, the normal force $F_{n}$ is determined by means of the penetration $p$ in normal direction, and the penetration velocity $\dot{p}$. A continuous contact force model with hysteresis damping according to [9] is implemented (Equation (8)). Nevertheless, selecting $n=1, m=0$ one can get the linear Kelvin-Voigt model, where the coefficients $c$ and $d$ are the spring and damping constants. Choosing $n=m$ a formulation according to [10] is obtained.

$$
F_{n}=c p^{n}+d p^{m} \dot{p}
$$

In order to calculate the friction forces without further discontinuous events, which would decrease the simulation speed and impede controller design, we use the continuously differentiable friction model of Makkar et al. [11]. They introduced the following function of the relative velocity $v_{r e l}$ to approximate the friction coefficient $\mu$ of the characteristic Stribeck curve.

$$
\begin{gathered}
\mu\left(v_{r e l}\right)=\gamma_{1}\left(\tanh \left(\gamma_{2} v_{r e l}\right)-\tanh \left(\gamma_{3} v_{r e l}\right)\right) \\
+\gamma_{4} \tanh \left(\gamma_{5} v_{r e l}\right)+\gamma_{6} v_{r e l}
\end{gathered}
$$

Thus, no ideal static friction can be obtained, because the actual force to be applied in the ideal static state is independent of the relative velocity $v_{\text {rel }}$ of the two contact points. Static friction is rather represented by sliding with very small relative velocities. However, this in fact matches the actual characteristics of many tribological systems [12]. To set the unknown, non-physical constants $\gamma_{i}(i=$ $1 \ldots 6)$ we use five parameters, which are shown in Figure 10. The parameters $\mu_{s}$ and $\mu_{k}$ denote the coefficients of static and kinetic friction. The limit velocities $v_{\varepsilon 1}$ and $v_{\varepsilon 2}$ define the beginning of mixed and viscous friction. The latter is described by the proportionality factor $k_{v}$.

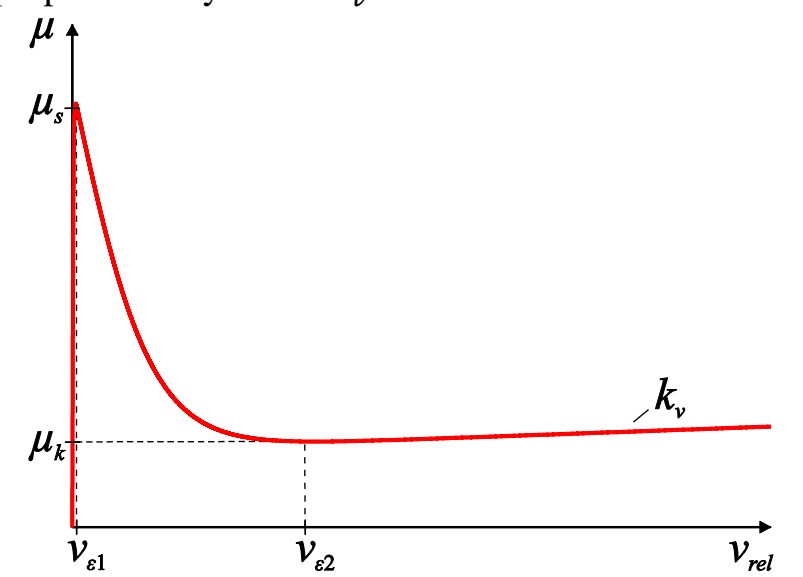

Figure 10: Approximation of the Stribeck friction curve
As an example, the following force vector (resolved in the LCS) is applied to the contact points of plane 1 of the rectangle-to-rectangle contact, if the contact condition is fulfilled.

$$
F_{\text {contact }}=\left(\begin{array}{c}
\mu_{x} \cdot F_{n} \\
F_{n} \\
\mu_{z} \cdot F_{n}
\end{array}\right)
$$

\section{Simulation Results}

In this section, we will show some of the obtained results. We present three experiments, which were performed in Dymola using the DASSL solver. The results are compared to a benchmark simulation in the commercial MBS software RecurDyn. Herein, a powerful recursive algorithm to model contact problems is implemented, which is based on contact forces as well [13]. Despite the comprehensive and complex solid-to-solid contact that can be used for arbitrary CAD-geometries, RecurDyn offers the possibility to utilize idealized contact definitions for simple surfaces. In each of the three experiments, all parameters ${ }^{2}$, including the contact force calculation as well as the solver settings, are attuned to fit each other exactly. The direction of gravity is the negative $y$-direction of the ICS. When selecting the presented experiments, we refer to the aforementioned surface configurations (c.f. Section 3).

Experiment 1 comprises a sphere $(r=0.015$, $m=0.11$ ) falling onto a cylindrical contact surface. The initial position of the sphere centroid is $(0,0.05,0.002)$. It is not coupled, whereas the position of the cylinder is fixed in the ICS. The latter has a diameter of $D=0.015$ and a length of $L=0.05$. In this configuration two collisions occur. After the second contact with the cylinder surface, the sphere falls down beside the cylinder. Figure 11 shows the $y$-position of the centroid of the sphere. One can see that the calculated trajectories of the different tools are comparable. In this case, the effect of the nonlinear damping seems to be slightly higher in Dymola than in RecurDyn. Nevertheless, the peaks in the normal forces nearly coincide at approximately $21 \mathrm{~N}$ and $9 \mathrm{~N}$ (not shown).

In Experiment 2, a cylindrical body $(d=0.015$, $l=0.03, \quad m=0.04)$ falls on a circular plane $(d=0.08)$ and then rolls down. The plane is fixed in the ICS, but rotated by $5^{\circ}$ around the $x$-axis. The initial position of the cylinder is $(0,0.02,0)$. As the cylinder reaches the end of the plane, the preliminary contact points are displaced along the length

\footnotetext{
${ }^{2}$ All units are specified according to the International System of Units (SI)
} 
direction. In Figure 12 some results are displayed exemplarily. We again observe that the positions of the centroid largely comply. The initial angular velocity (around the length direction) of the cylinder after the hit is slightly smaller in RecurDyn than in Dymola, while the acceleration is equivalent. The RecurDyn model (solid-to-solid contact) shows some implausible behavior right before the end of the plane is reached. Unless no external force is applied, deceleration can be monitored here. For these two reasons, the cylinder stays about $10 \mathrm{~ms}$ longer on top of the plane.

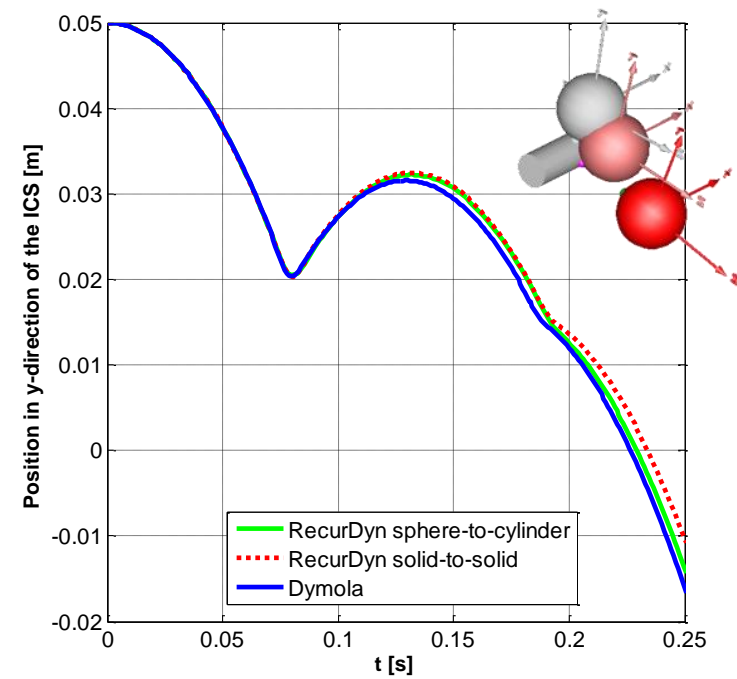

Figure 11: Sphere falling on a fixed cylinder; parameters: $c=1 e^{5}, d=10, n=1.5, m=1.5$, $t=5 e^{-3}, \quad \mu_{s}=0.03, \quad \mu_{k}=0.02, \quad v_{\varepsilon 1}=0.01$, $v_{\varepsilon 2}=0.1, k_{v}=0$

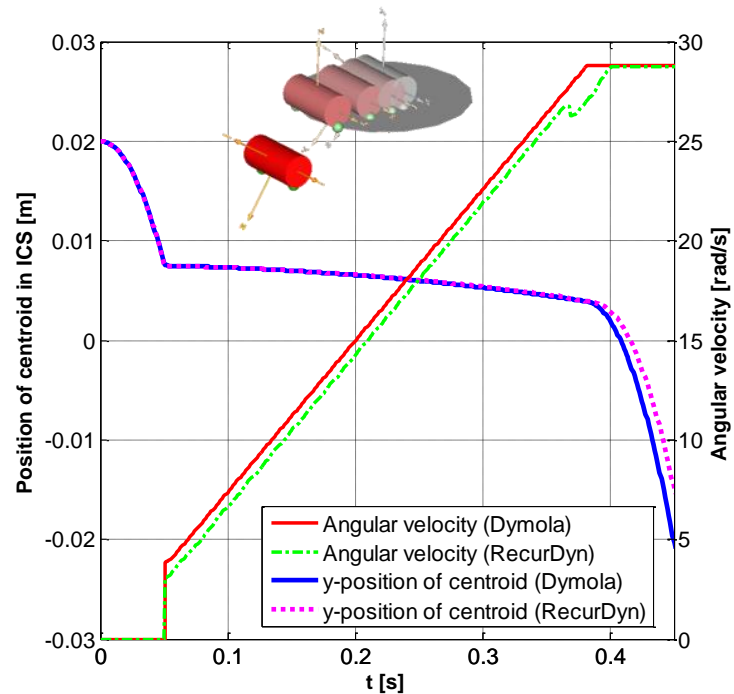

Figure 12: Cylinder on a circular plane; parameters: $c=5 e^{3}, d=5 e^{2}, \quad n=1, \quad m=0, \quad t=3 e^{-3}$, $\mu_{s, k}=0.12, v_{\varepsilon 1,2}=0.01, k_{v}=0$

Experiment 3 contains a cuboid body $(l=0.07$, $w=0.1, \quad h=0.05, \quad m=2.75) \quad$ falling and afterwards sliding on a rectangular plane. The plane $(l=0.2, w=0.25)$ is again fixed in the ICS and rotated by $15^{\circ}$ around the $z$-axis. What can be seen in the plots (Figure 13) is that we get very similar movement of the box, despite an observed higher velocity in RecurDyn. In our opinion, the small divergence we obtain may be due to three reasons. (1) Again, the effect of the damping is slightly higher in Dymola, which leads to more sliding and therefore more decelerating friction forces. (2) The approximation of the Stribeck curve varies, which may lead to differences. (3) The handling of forces at the edge of the plane may be different (compare to Experiment 2).

In the case of our contact model the contact points are moved as displayed in Figure 14 (see Figure 8 also). As $b_{13}$ constitutes the first edge of the cube to reach the end of the plane, it is the first contact point to be split up and moved in the length and width direction. The movement is limited to the respective dimensions of the cube.

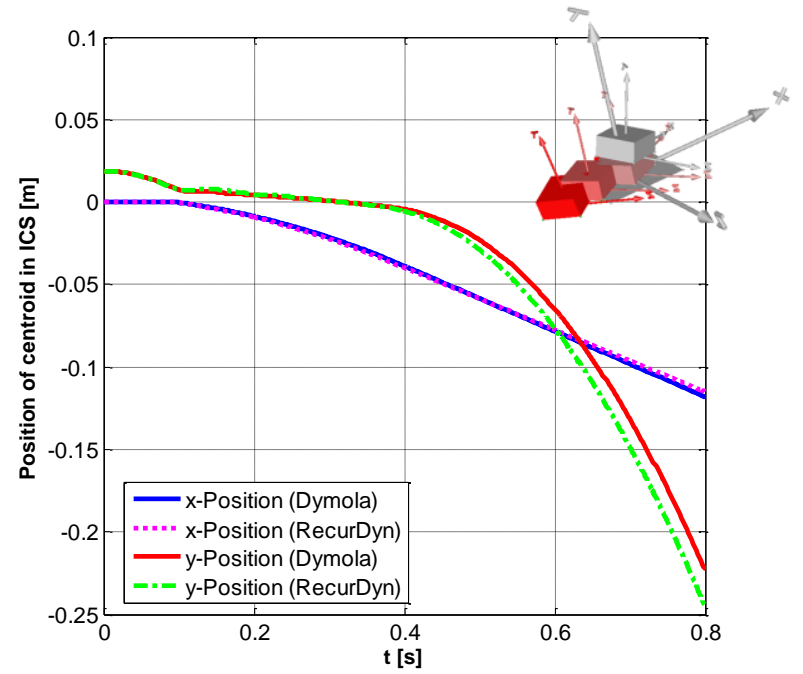

Figure 13: Box falling and sliding on a plane; parameters: $c=1 e^{7}, d=1 e^{7}, n=1.1, \quad m=1$, $t=5 e^{-3}, \quad \mu_{k}=0.08, \quad \mu_{s}=0.12, \quad v_{\varepsilon 1}=1 e^{-4}$ $v_{\varepsilon 2}=1 e^{-2}, k_{v}=0$

To evaluate the efficiency of the contact library in the context that was outlined in the beginning, we also investigated the "CPU-time for integration". Table 1 compares this characteristic property of the aforementioned models (simulated time $t=1 \mathrm{~s}$ ). The results depict the experiences we made in various tests and further experiments. While in general, the integration times are comparable, they strongly depend on the number of contact points in Dymola. One can observe a strong increase, especially when movement of contact points occurs and the calculated displacement is filtered. On the other hand, modeling a rolling body sometimes leads to problems in RecurDyn, which is reflected in the CPU-time (see also Figure 12). 


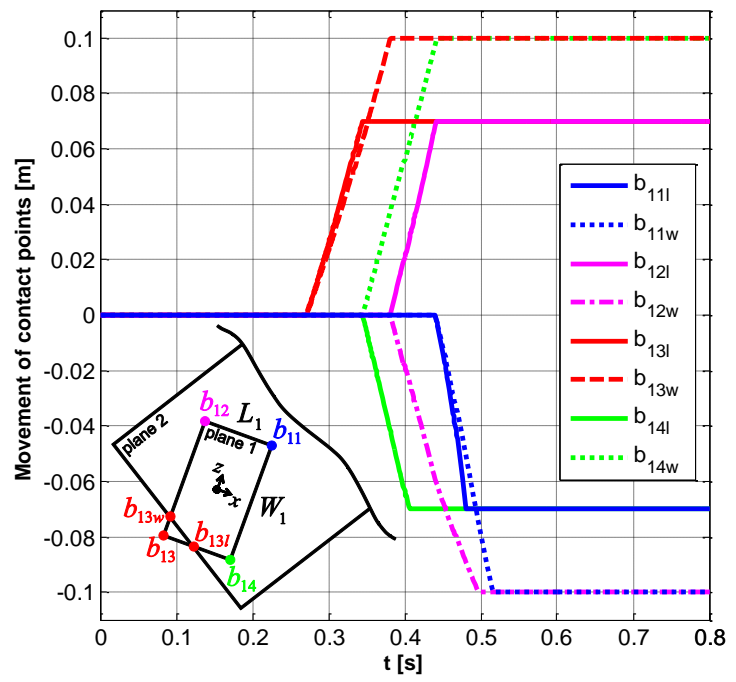

Figure 14: Contact point movement resolved in the local coordinate systems

Table 1: CPU-time for integration ${ }^{3}$

\begin{tabular}{|c|c|c|}
\hline & Dymola & RecurDyn \\
\hline Experiment 1 & $0.37 \mathrm{~s}$ & $\begin{array}{l}0.20 \mathrm{~s} \text { (sphere-to-cylinder) } \\
0.50 \text { s (solid-to-solid) }\end{array}$ \\
\hline Experiment 2 & $\begin{array}{l}3.52 \mathrm{~s} \text { (filter } 10 \mathrm{kHz}) \\
0.61 \mathrm{~s} \text { (filter disabled) } \\
\end{array}$ & 4.98s (solid-to-solid) \\
\hline Experiment 3 & $\begin{array}{l}5.86 \mathrm{~s} \text { (filter } 10 \mathrm{kHz}) \\
5.63 \mathrm{~s} \text { (filter disabled) }\end{array}$ & $\begin{array}{l}0.59 \mathrm{~s} \text { (surface-to-surface) } \\
1.90 \mathrm{~s} \text { (solid-to-solid) }\end{array}$ \\
\hline
\end{tabular}

\section{Conclusion and Future Work}

In conclusion, we state that our idealized contact library provides a powerful and easy to use opportunity to model contact phenomena of simple contact geometries. Results of various experiments where compared and verified by means of analogous simulations in RecurDyn. The chosen architecture with the implemented contact interface and the configurable contact block matches the idea of reusing composable models. It is therefore especially useful in the conceptual design of mechatronic systems.

In the future, we want to provide more contact surfaces. In addition, we will investigate a possibility to save simulation time by disabling the contact calculation when they are not needed. Despite the validation with RecurDyn, comprehensive measurements are necessary. The major drawback of the force-based approaches is that the spring/damper parameters cannot be obtained directly from the given material properties. These parameters also depend on the surface combination and are usually determined experimentally. To tackle this, we plan to

\footnotetext{
${ }^{3}$ The simulations were performed on an Intel Core2Duo CPU with $2.53 \mathrm{GHz}$ and 4GB RAM
}

provide ready-to-use parameter sets for often-used combinations. After making our library publicly available, we also hope to identify further opportunities for improvement with the help of the Modelica community.

\section{Acknowledgements}

This work was developed in the project "ENTIME: Entwurfstechnik Intelligente Mechatronik" (Design Methods for Intelligent Mechatronic Systems). The ENTIME project was funded by the state of North Rhine-Westphalia (NRW), Germany, and the EUROPEAN UNION, European Regional Development Fund, "Investing in your future".

In addition, the authors would like to thank the reviewers for reading the text carefully and giving useful suggestions on where to improve both the paper and the library.

\section{References}

[1] F. Bauer, J. Gausemeier, D. Köchling, F. Oestersötebier: Approach for an Early Validation of Mechatronic Systems using Idealized Simulation Models within the Conceptual Design. In: Proceedings of the 5th CIRP Conference on Industrial ProductService Systems, Bochum, March 14-15, 2013

[2] T. Juhász. Advanced Solutions in ObjectOriented Mechatronic Simulation. Ph.D. Thesis, Dept. of Control Engineering and Information Technology, Budapest University of Technology and Economics, 2008

[3] M. Otter, H. Elmqvist, J. Díaz López. Collision Handling for the Modelica MultiBody Library. In: Proceedings of the 4th International Modelica Conference, Hamburg, March 7-8, 2005

[4] G. Hippmann. Modellierung von Kontakten komplex geformter Körper in der Mehrkörperdynamik. Ph.D. Thesis, Vienna University of Technology, 2004

[5] K. L. Johnson. Contact Mechanics. Cambridge University Press, Cambridge, 1985

[6] J. J. Kalker. Three-dimensional elastic bodies in rolling contact. Kluwer Academic Publishers, Norwell, 1990

[7] W. Sextro. Dynamical contact problems with friction. Springer-Verlag, Berlin, Heidelberg, 2007

[8] D. W. Marhefka, D. E. Orin. A Compliant Contact Model with Nonlinear Damping for Simulation of Robotic Systems. IEEE 
Transactions on Systems, Man, and Cybernetics, 29(6), 1999

[9] H. M. Lankarani, P. E. Nikravesh. Continuous Contact Force Models for Impact Analysis in Multibody Systems. Nonlinear Dynamics, 5, 1994

[10] K. H. Hunt, F. R. E. Crossley. Coefficient of restitution interpreted as damping in vibroimpact. ASME J. Appl. Mech, 1975

[11] C. Makkar, W. E. Dixon, W. G. Sawyer, G. Hu. A New Continuously Differentiable Friction Model for Control Systems Design. In: Proceedings of the 2005 IEEE/ASME International Conference on Advanced Intelligent Mechatronics, Monterey CA, July, 2005

[12] V.L. Popov. Kontaktmechanik und Reibung. Springer-Verlag, Berlin, Heidelberg, 2009

[13] B. Roh, H. Aum, D. Bae, H. Cho, H. Sung. A Relative Contact Formulation for Multibody System Dynamics. KSME International Journal, 14(12): 1328-1336, 2000 